\title{
РАЗРАБОТКА И ИСПОЛЬЗОВАНИЕ ЛОГИЧЕСКОЙ СХЕМЫ ДЛЯ РАСЧЁТА НАДЁЖНОСТИ СИСТЕМ АПК
}

\author{
Пряхин В.Н. \\ ГБОУ ВО МО «Университет «Дубна», д.т.н., профессор \\ Ильинич Олег Витальевич \\ Лукина Е.C. \\ ГБОУ ВО МО «Университет «Дубна», ассистент
}

\begin{abstract}
Аннотация. Проведён анализ моделей процессов, происходящих в сложных системах на объектах АПК. Произведена оценка эффективности и оптимизации сложных систем различного назначения. Обосновано применение в научных исследованиях универсальной имитационной модели, которая может быть адаптирована к любому объекту АПК из указанного класса. Проанализированы различные математические схемы систем автоматического управления и систем массового обслуживания. Предложен подход к составлению логической схемы для оценки надёжности технической системы, на основе которого формируются формулы расчёта вероятности их надёжного функционирования.

Annotation. The analysis of models of processes occurring in complex systems at agricultural facilities is carried out. The efficiency and optimization of complex systems for various purposes are evaluated. The use of a universal simulation model in scientific research, which can be adapted to any object of the agro-industrial complex from the specified class, is justified. Various mathematical schemes of automatic control systems and queuing systems are analyzed. An approach to drawing up a logical scheme for assessing the reliability of a technical system is proposed, on the basis of which formulas for calculating the probability of their reliable functioning are formed.

Ключевые слова: Статистический анализ; система массового обслуживания; техническая система; логическая схема; вероятность безопасного функционирования.

Keywords: Statistical analysis; queuing system; technical system; logical scheme; probability of safe operation.

\section{ВВЕДЕНИЕ}

При построении моделей процессов, происходящих в сложных системах на объектах агропромышленного комплекса (АПК) с целью оценки эффективности и оптимизации этих систем используются различные математические схемы:

- статистический анализ систем управления;

- конечные и вероятностные автоматы;

- марковские случайные процессы;

- дифференциальные и конечно-разностные уравнения;

- системы массового обслуживания (СМО); и др.

При этом для исследования различных сложных систем (животноводческих комплексов, тепличных комбинатов, транспортных систем и др.) необходимо строить разнообразные модели и их системы [1].

Следует отметить, что современные методы подготовки задач позволяют строить так называемые универсальные имитационные модели, способные настраиваться на любой объект из указанного класса.

В этом случае используется универсальная имитационная программа, составляемая заранее для некоторой стандартной формы сложной системы, охватывающей широкий класс реальных объектов, а также комплекс программ преобразования входной и выходной информации с немашинных языков, привычных для исследователей, к стандартной форме, принятой при составлении программ.

При этом исследователь вводит информацию и получает результаты в привычных для себя терминах, соответствующих его математическим и содержательным представлениям, а исследования проводятся с помощью автоматизированной имитационной модели.

Тогда схема даёт существенную экономию времени разработки машинных моделей и повышает возможности исследователей пользоваться совершенным аппаратом, разработка которого под силу только крупным научно-исследовательским коллективам [2].

ОСНОВНАЯ ЧАСТЬ

В процессе различного рода научных исследований часто приходится использовать подход к составлению логической схемы для оценки надёжности технической системы (ТC), на основе которого формируются формулы расчёта вероятности их надёжного функционирования.

Как правило, перед расчётом надёжности системы проводится предварительная работа по составлению логической схемы расчёта.

Эта работа состоит из 3 основных этапов:

- описание работы системы;

- классификация отказов элементов и систем;
\end{abstract}


- $\quad$ составление структурной (логической) модели безотказной работы системы.

При этом для каждого блока системы расчёт функции надёжности ведётся по выбранной методике расчёта. Все выделенные блоки нумеруются или обозначаются буквой.

Затем перечисляются комбинации блоков, обеспечивающих безотказную работу системы.

Эти комбинации могут быть составлены в виде таблицы:

Таблица 1.

\begin{tabular}{|c|c|}
\hline № комбинации & Для безотказной работы системы необходимо, чтобы были исправны блоки \\
\hline 1 & $\mathrm{~A}$, Б, В, Г, Д, Е \\
\hline 2 & $\mathrm{~A}$, Б, К, Г, Д, Е \\
\hline 3 & $\mathrm{~T}, \mathrm{M}$, П, Г, Д, Е \\
\hline 4 & $\mathrm{~T}, \mathrm{M}, \mathrm{C}$, Г, Д, Е \\
\hline
\end{tabular}

На основании табл. 1 составляется словесная формула:

«Безопасная работа системы обеспечивается, если исправны блоки: А и Б и В (или К) или Т и М (или С) и Ги Д и Е».

Вместо этой словесной формулы иногда более удобно составить логическую схему (рис. 1), где слову «и» соответствует последовательное соединение блоков, а слову «или» соответствует параллельное соединение блоков [3].

При этом речь идёт не о функциональной схеме, а о логической схеме для расчёта надёжности.

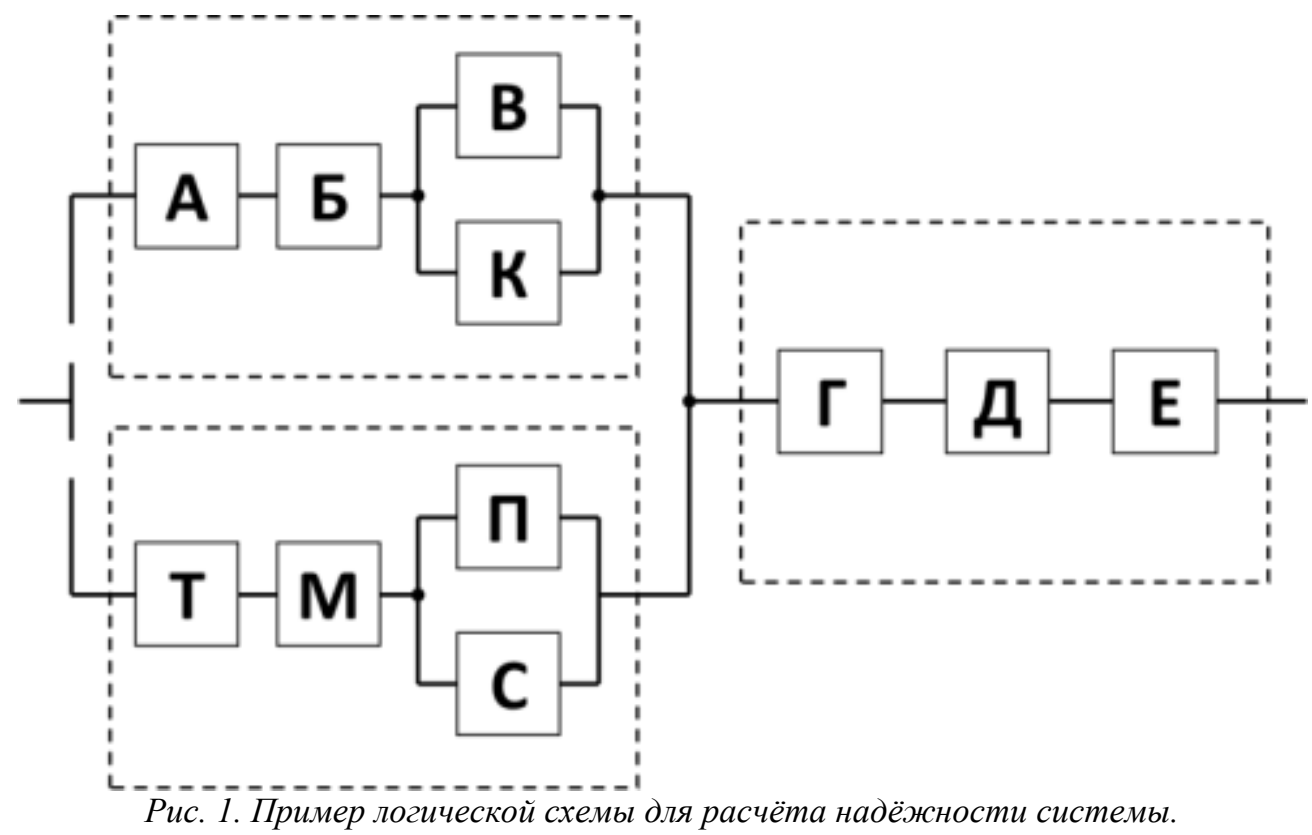

По приведённой логической схеме (рис.1) или словесной формуле можно составить зависимость функции надёжности системы от функций надёжности блоков.

Для системы, имеющей изображённую на рис.1 логическую схему расчёта, вероятность безотказного функционирования будет:

$$
\mathrm{P}=[1-(1-\mathrm{P} 1) *(1-\mathrm{P} 2)] * \mathrm{P} 3
$$

где P1, P2, Р3 - вероятности безотказного функционирования в течение заданного времени $\mathrm{t}$ соответствующих групп блоков, отмеченных на рис.1 пунктиром.

Тогда вероятность безотказной работы первой группы блоков:

$$
\mathrm{P}_{1}=\mathrm{P}_{\mathrm{A}} * \mathrm{P}_{\mathrm{B}} *\left[1-\left(1-\mathrm{P}_{\mathrm{B}}\right) *\left(1-\mathrm{P}_{\mathrm{K}}\right)\right]_{*}
$$

Для второй группы:

$$
\mathrm{P}_{2}=\mathrm{P}_{\mathrm{T}} * \mathrm{P}_{\mathrm{M}} *\left[1-\left(1-\mathrm{P}_{\Pi}\right) *\left(1-\mathrm{P}_{\mathrm{C}}\right)\right] *
$$


Для третьей группы:

$$
P_{a}=P_{\Gamma} * P_{\mathbb{Z}} * P_{E^{x}}
$$

Подставив эти выражения для групп блоков в формулу (1), получим функцию надежности системы:

$$
\begin{aligned}
& \mathrm{P}(t)=1-\left\{1-\mathrm{P}_{A}(t) * \mathrm{P}_{\mathrm{B}}(t) *\left[1-\left(1-\mathrm{P}_{\mathrm{B}}(t)\right) *\left(1-\mathrm{P}_{\mathrm{K}}(t)\right)\right]\right\} *\left\{1-\mathrm{P}_{\mathrm{T}}(t) * \mathrm{P}_{\mathrm{M}}(t) *\left[1-\left(1-\mathrm{P}_{\mathrm{M}}(t)\right) *\right.\right. \\
& \left.\left.\left(1-\mathrm{P}_{\mathrm{C}}(t)\right)\right]\right\} * \mathrm{P}_{\Gamma}(t) * \mathrm{P}_{\mathrm{Z}}(t) * \mathrm{P}_{\mathrm{E}}(t)
\end{aligned}
$$

На рис.1 представлена общая схема и соответствующая ей формула 5.

Рассмотрим техническую систему и расчет вероятности безотказного функционирования подводных переходов магистральных газопроводов (ППМГ).

Огромные размеры территории, удалённость основных районов добычи углеводородов от потребителя, высокие объёмы экспорта - определяющие факторы нефте- и газотранспортной сети России. Особенность территории страны - густая сеть рек и других водных объектов, являющихся наиболее сложными и опасными участками трубопроводов.

При этом количество подводных переходов магистральных газопроводов (ППМГ), их значимость для безопасности жизнедеятельности населения обусловили рассмотрение вопросов эксплуатации и строительства как отдельного специфического водопользователя, во многом определяющего правила использования водных ресурсов в природообустройстве прилегающих ландшафтов.

Тогда ППМГ через водные объекты являются весьма специфическими инженерными сооружениями и сложными природно-техническими комплексами.

В условиях различного типа водных преград эксплуатация магистральных трубопроводов сопряжена с многофакторными рисками их повреждения, несущими угрозу экологической безопасности природных комплексов и значительные технические риски, связанные с необходимостью бесперебойной поставки углеводородного сырья потребителю.

В этой связи, является необходимой оценка релевантности проводимых наблюдений и их статистических обобщений в рамках мониторинга магистральных газопроводов и факторов, влияющих на их состояние.

Многолетний опыт эксплуатации ППМГ показывает, что среди основных технологических факторов влияющих на появление неисправных ниток трубопроводов являются давление газа в трубе и ее диаметр.

Приведем пример расчета надежности системы на реальных данных отрасли, полученных в результате обследования ППМГ в июне 2009 года.

Согласно результатам проведенных обследований, каждый из приведенных факторов можно условно разделить на два диапазона: давление газа до 75 атм. и свыше 75 атм.; диаметр трубы меньше 1020 мм. и больше 1020 мм.

Введем следующие обозначения:

$\mathrm{P}_{1}$ - вероятность неисправной работы объекта в зависимости от давления газа; $\mathrm{P}_{2}$ - вероятность неисправной работы объекта в зависимости от диаметра трубопровода.

Разделим каждый фактор по диапазонам:

$\mathrm{P}_{1}^{1}$ - вероятность неисправной работы объекта при давлении газа до 75 атм.;

$\mathrm{P}_{1}^{\frac{1}{2}}$ - вероятность неисправной работы при давлении газа не меньше 75 атм.;

$\mathrm{P}_{2}^{1}$ - вероятность неисправной работы объекта при диаметре трубопровода меньше 1020 мм;

$\mathrm{P}_{2}^{2}$ - вероятность неисправной работы при диаметре трубопровода не меньше 1020 мм;

Согласно статистическим исследованиям отрасли имеем следующие принятые вероятности соответствующих событий:

$P_{1}^{1}=0,32 ; P_{1}^{2}=0,24 ; P_{2}^{11}=0,26 ; P_{2}^{2}=0,23$.

Функция вероятности выхода системы в отказ: $\mathrm{P}=\mathrm{P}_{1} * \mathrm{P}_{2}$.

$$
\begin{aligned}
& P_{1}=\left[1-\left(1-P_{1}^{1}\right) *\left(1-P_{1}^{2}\right)\right]=[1-(1-0,32) *(1-0,24)]=0,48 ; \\
& P_{2}=\left[1-\left(1-P_{2}^{1}\right) *\left(1-P_{2}^{2}\right)\right]=[1-(1-0,26) *(1-0,23)]=0,43 ;
\end{aligned}
$$




$$
P=P_{1}+P_{2}=0,48+0,43=0,21 \text {. }
$$

Следует отметить, что в практике эксплуатации ППМГ при исследовании надежности объектов важнейшую роль играет сбор и обработка информации о надежности ТС, представляющие собой совокупность организационно-технических мероприятий по получению необходимых достоверных сведений о надежности исследуемых объектов.

При этом сбор и обработку информации о надежности объектов выполняют с целью [4]: усовершенствования конструкции, технологии изготовления, сборки и испытаний объектов, обеспечивающих повышение надежности:

- разработки мероприятий по совершенствованию диагностирования, технического обслуживания, повышения качества текущего и капитального ремонтов и снижение затрат на их проведение;

- оптимизации норм расхода запасных частей.

На рис.2 представлены основные задачи системы сбора и обработки информации (ССОИ).

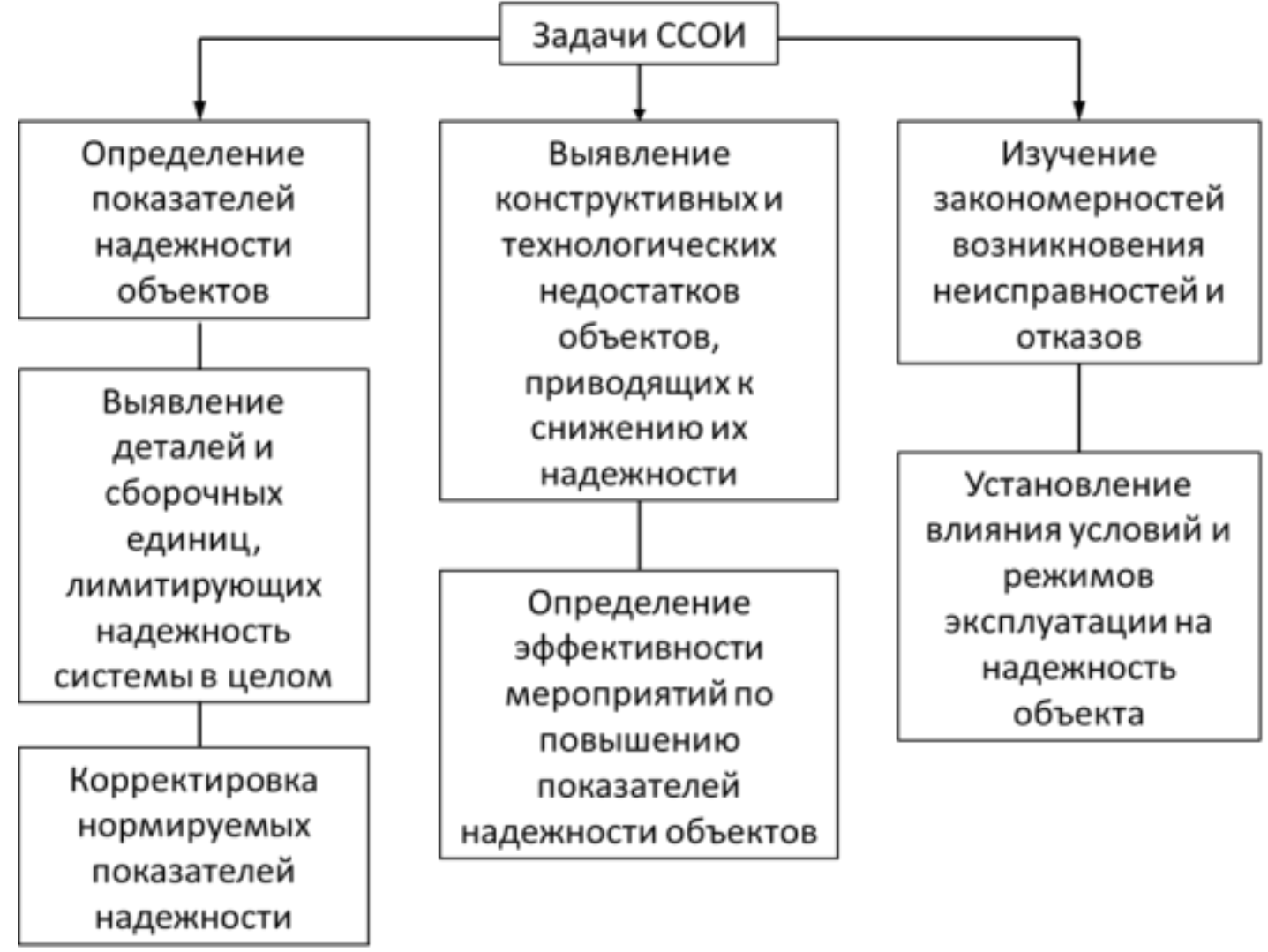

Рис. 2 Основные задачи ССОИ на технических объектах

Важным источником информации о надежности в гарантийный период эксплуатации объекта служат рекламации от потребителей ТС.

При этом информация о надежности объекта должна быть:

- д достоверной (истинной, правильной, отражающей объективные факторы без домыслов и догадок);

- полной (исчерпывающей, содержащей все существенные сведения, которые учитывают во время принятия решений);

- однородной (относящейся к одинаковым объектам, эксплуатирующимся примерно в одинаковых условиях);

- дискретной (разделена по отдельным признакам);

- своевременной (могла использоваться для изменения конструкций, корректировки технологического процесса изготовления, ремонта машины и технического обслуживания).

Следует отметить, что сбор, обработка и анализ информации о надежности ТС связаны с необходимостью исследования случайных событий и величин. 
Кроме того, все показатели надежности относятся к категории случайных величин, которые рассчитывают методами теории вероятностей и математической статистики [6,7].

Особый интерес, на наш взгляд, представляют вопросы совершенствования методов оценки надёжности и безопасности технических систем (ТС) в связи с задачами повышения значений этих показателей, как в промышленном, так и в с.-х. производстве.

При этом основной целью является разработка логической модели надёжности ТС и соответствующих логических методов оценки надёжности систем, которые бы использовали не только традиционные вероятностные показатели надёжности, но и детерминированные логические показатели.

В этом случае, задача оценки надёжности ТС сводится к построению её математической модели в виде логических функций, которые выражают моменты её последовательных отказов и восстановлений через аналогичные моменты всех элементов данной ТС.

Другими словами, решение поставленной задачи решается путём построения адекватной логической модели надёжности ТС, позволяющей свести оценку надёжности системы к вычислению её логических функций надёжности [8, 9].

Такой подход хорошо согласуется с решением аналогичных задач и при разработке различных автоматизированных систем, эксплуатируемых на объектах АПК.

ВЫВОДЫ

- При оценке надежности работы ППМГ вполне возможно и рационально использовать общие логические схемы для расчета вероятности безотказного функционирования ТС;

- Для удобства сбора и систематизации исходных данных, а также для проведения автоматизированных вероятностных расчетов рентабельно применять автоматизированную систему управления технологическими процессами производства;

- Обосновано применение в научных исследованиях универсальной имитационной модели, способной настраиваться на любой объект АПК;

- Предложенный нами подход к разработке и использованию логической системы для расчета надежности систем может быть использован в промышленном и с.-х. производстве и учитывает специфику объектов АПК, функционирующих в различных условиях испытаний.

\section{ЛИТЕРАТУРА}

Пряхин В. Н., Соколов В. В., Ильинич О. В. Анализ различных вариантов автоматизированных систем управления поливом, подкормкой и увлажнением воздуха в условиях открытого и защищенного грунта // Международный технико-экономический журнал. - М., 2010. - № 5. - С. 48-50.

Пряхин В.Н., Карапетян М. А., Крюков Ю. А. Совершенствование технических средств и технологических процессов сельскохозяйственного производства: монография / В. Н. Пряхин, М. А. Карапетян, Ю. А. Крюков. Москва: Изд-во «Спутник +», 2021. - 258 с.

Пряхин В.Н., Гайдукевич С.В., Ильинич О.В. Разработка логической схемы для расчета надежности систем на объектах народного хозяйства. // Международный научный жкрнал. -М., 2011, №2. - С. 72-75.

Пучин Е.А., Дидманидзе О.Н., Лезин П.П. и др. Надежность технических систем. - М.: УМЦ «Триада», 2005. -353 c.

Ильинич О. В. Анализ системы мониторинга состояний подводных переходов магистральных газопроводов / О. В. Ильинич. - Электрон. текстовые дан. // Природообустройство, 2011. - Вып. 3 - С. 39-42.

Шведов А.С. Теория вероятностей и математическая статистика: учебное пособие для вузов. 2-ое изд., перераб. и доп. - М.: Изд. Дом ГУ ВШЭ, 2005. - 254c.

Пряхин В.Н. Безопасность жизнедеятельности: Курс лекций. - М.: 000 «Мегаполис», 2019. - 115с.

Левин В.И. Логические методы расчета надежности систем. // Системы управления, связи и безопасности, №2, 2017. - С. 182-195.

Лукьянов В.С., Кузнецова Е.С. Курс лекций по дисциплине «Надежность АСОИУ»: учебное пособие. Волгоград, 2007. - 99с. 